\title{
New concepts in the appraisal and management of birdshot retinochoroiditis, a global perspective
}

\author{
Marina Papadia • Carl P. Herbort Jr.
}

Received: 5 October 2014/ Accepted: 27 January 2015/Published online: 14 February 2015

(C) Springer Science+Business Media Dordrecht 2015

\begin{abstract}
Birdshot retinochoroiditis (BRC) is a relatively recently described entity. BRC uveitis is predominant in the posterior segment with dual, independent retinal, and choroidal inflammation. The disease has no known extra-ocular inflammation sites and yet features the strongest known HLA association: HLA-A29 is present in close to $100 \%$ of cases. Aim in this mini-review was to readjust the appraisal of BRC in the light of a global approach including the full array of investigational procedures. Historical background and the genesis of the disease name were searched. Global disease description including both the retina and the choroid was given. Retinal involvement was clearly characterized, pointing toward the profuse leakage of retinal vessels of all sizes in early disease and widespread atrophy in under treated patients. The importance of exploration of choroidal disease, unavailable until the early 1990s before the
\end{abstract}

M. Papadia · C. P. Herbort Jr. ( $\square)$

Retinal and Inflammatory Eye Disease, Centre for Ophthalmic Specialized Care (COS), Clinic Montchoisi, Rue Charles-Monnard 6, 1003 Lausanne, Switzerland e-mail: carl.herb@bluewin.ch

M. Papadia

e-mail: marinapapadia@yahoo.com

M. Papadia

Ospedale Padre Antero Micone Genova, Genova, Italy

C. P. Herbort Jr.

University of Lausanne, Lausanne, Switzerland advent of indocyanine green angiography (ICGA) was stressed, allowing early diagnosis of disease. Despite its proven importance to explore the choroid, ICGA is still sparsely used. Existing diagnostic criteria were found to be clearly inappropriate not allowing early diagnosis and are in need to be revised, taking into account both retinal and choroidal aspects of the disease, in order to make early diagnosis possible and hence allow proper management .

Keywords Birdshot retinochoroiditis - Indocyanine green angiography - Optical coherence tomography . Visual field · Early diagnosis of birdshot retinochoroiditis - Clinical phenotype

\section{Introduction}

The purpose of this review on birdshot retinochoroiditis (BRC) was to point out incongruities, omissions, and misconceptions in the approach of the disease since it was first described in 1980-1981. Reappraisal of the disease was presented, taking into account all investigational procedures at our disposal to foster a global, comprehensive, and precise approach to BRC.

Without being an exhaustive review, the main points addressed include disease history, terminology and disease name, disease semiology and disease process, diagnostic steps and functional testing including fundus photography, fluorescein angiography (FA), 
indocyanine green angiography (ICGA), optical coherence tomography (OCT), automated perimetry, electrophysiology, and HLA typing. Furthermore, adequate diagnostic criteria were put forward allowing early diagnosis and the need for appropriate management and monitoring of evolution was stressed. Discrepancies versus the commonly used approach were highlighted.

\section{Historical aspects}

Birdshot retinochoroiditis is a rare, bilateral, retinochoroidal, inflammatory disease without known systemic involvement; it is mostly seen in Caucasians [1, 2]. In 1980 Ryan and Maumenee first described the bilateral retinochoroidal inflammatory disease and gave it the name of birdshot retinochoroidopathy, as they found that lesions had a comparable pattern seen in wounds produced by birdshot shotguns [2]. In 1981, Gass described 11 similar cases and called the disease vitiliginous chorioretinitis [3].

No known systemic association has been found so far for this disease.

Both reports described keratic precipitates (KPs) and/or mutton-fat precipitates on the posterior surface of the detached vitreous. This fact was never mentioned in subsequent series and became an exclusion criterion in the research diagnostic criteria decided upon in a consensus workshop on BRC held in 2002 and published in 2006 [4]. An important FA sign that Gass observed was the loss of fluorescein into the retina and the weak fluorescein coloration of large veins in the venous phase of the angiographic cycle, speaking of an increased retinal circulation time, a finding that we have been able to explain using dual FA/ICGA (see below) [5]. He correctly attributed the depigmented areas to the loss of choroidal stromal melanocytes. He also contributed to the belief that corticosteroids were of little efficacy. The subsequent ground breaking article, published by Nussenblatt et al. in 1982, associated HLA-A29 antigen with the disease [6]. However, the article that gave determining information on the pathophysiology of the disease, published by Gaudio et al. showed that choroidal birdshot lesions consisted of infiltrates surrounding choroidal stromal melanocytes and that they were of partial thickness, not touching the choriocapillaris/ retinal pigment epithelium complex or the sclera; and contained epithelioid cells [7].

\section{Terminology (genesis of the disease name)}

In their inaugural article, Ryan and Maumenee chose the name of birdshot retinochoroidopathy. Oddly the term birdshot was chosen in reference to the aspect of shotgun wounds. In the second component of the name, retinochoroidopathy, they chose to put retina in front of choroid [2]. In his article, Gass gave the name of vitiliginous chorioretinitis to the disease, placing "choroid" first [3]. In both articles, the choroidal involvement was at the origin of the first term of the eponym as both "birdshot" and "vitiliginous" refer to the rice-shaped, depigmented choroidal fundus lesions that characterize the condition. Today, there is no question that of the two terms, "birdshot" is the universally used and accepted term. It is unfortunate that the condition was called a retinochoroidopathy in the first published article, although the authors clearly acknowledged its inflammatory character [2]. The inflammatory character appears more clearly in the name chosen by Gass, as he speaks of vitiliginous chorioretinitis. It is now clear that BRC is not a retinochoroidopathy; the suffix "pathy" indicates a non-inflammatory condition, as is the case for central serous chorioretinopathy. As the nature of the condition is clearly inflammatory, obviously the suffix "itis" should be used. The last question regarding the denomination is the choice of the second term of the eponym. Should "retino-" precede "choroid" or vice versa, a point on which the two initial articles were contradictory $[2,3]$. In full-blown or late disease, the clinical signs that strike the clinician most are the depigmented lesions that led to the terms "vitiliginous" and "birdshot". The striking choroidal lesions pushed some authors to also to give precedence to the choroid over the retina in the second term of the eponym, speaking of chorioretinitis or chorioretinopathy. It is our opinion that attention should be given in the second part of the name to the retinal disease that, in the early exudative phase of the disease causes functional loss and in the late phase evolves toward severe atrophy. The retina is the structure at the origin of most of the disease morbidity. In contrast, the choroidal lesions remain occult and are only detectable using ICGA in the early phase of disease and even in the late phase of the disease when depigmented BRC lesions are strikingly visible they cause minimal morbidity. For both these reasons the disease should be called "birdshot retinochoroiditis" rather than the other way around. 


\section{Disease semiology \& disease process}

Disease presentation

BRC is an intraocular inflammatory condition without any known systemic associations. It involves both the retina and the choroid and is linked in nearly all cases to the presence of the HLA-A29 human leucocyte histocompatibility antigen. The disease occurs almost exclusively in Caucasians and affects patients in their $50 \mathrm{~s}$ with preponderance for female patients, which is nearly the double of male patients.

\section{Anterior segment: BRC is a uveitis} of the granulomatous type

Anterior segment inflammation is mild to subclinical and frank anterior chamber inflammation is rare. In a collective of 13 patients (26 eyes) where measurements were available at presentation, laser flare photometry revealed a mean flare value of $10.6 \mathrm{ph} /$ $\mathrm{ms}$ in treatment-naïve patients [8]. This finding indicates that there is minimal anterior spillover inflammation in this posterior uveitis and therefore laser flare photometry is not suitable for monitoring disease activity in most cases [9]. However, since the early reports by both Ryan and Maumenee and Gass, KPs were no longer described, even leading a group of experts to list the presence of KPs as an exclusion criterion [4]. We found small granulomatous KPs in 3/19 (16\%) treatment-naïve BRC patients (Fig. 1) [10]. One single granulomatous KP was detected in two of these patients, a feature also described by Gass in his first report. This very discrete occurrence of KPs might be the reason why in subsequent reports the presence of KPs was overlooked; indeed a series of at least 8-9 treatment-naïve patients is needed to have one case that may show only one KP. It is therefore puzzling that the presence of KPs was not mentioned in a study of 80 BRC patients examined at baseline [11]. It should be noted here that the term granulomatous uveitis is not used in its histological sense but is a clinical term based on a set clinical signs that were classically associated with granulomatous diseases, including, among others, clearly identifiable KPs (from small to mutton-fat). This term is in fact a misnomer as it is a clinical description for which a histo-pathological term is used.

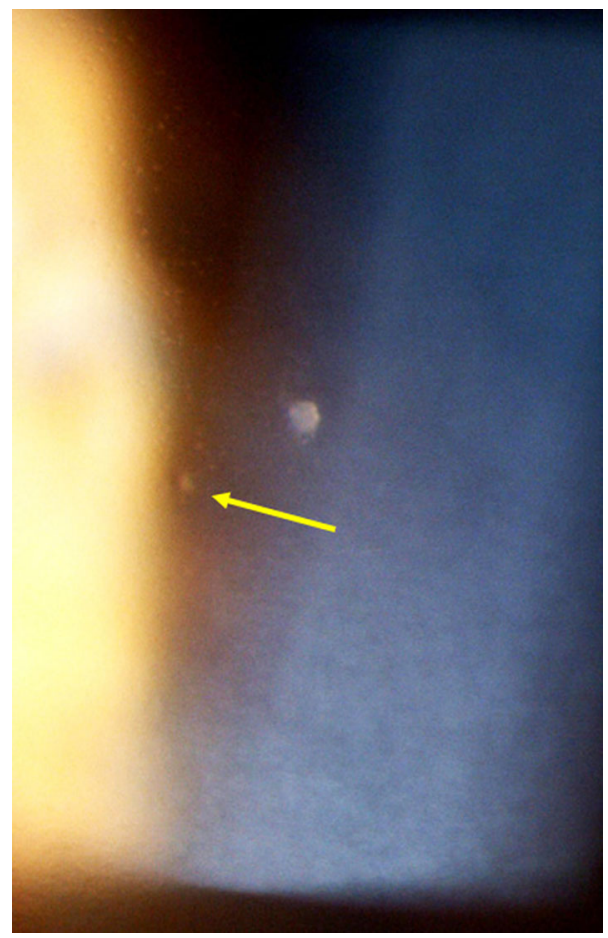

Fig. 1 Granulomatous keratic precipitates (KPs) in a treatment-naïve BRC patient. Disease had been evolving without treatment for 10 years when this single granulomatous KP was observed. A second smaller KP is visible to the left of the medium-sized KP (arrow)

\section{Vitreous}

All reports indicate unanimously that inflammation in this compartment always shows cells, giving the fuzzy fundus observed in most treatment-naïve patients. Vitritis is a constant feature in BRC. Ryan and Maumenee describe debris and opacities in all of their patients, whereas Gass clearly described cells in all 11 of his patients, of which one had $1+$ cells, six had $2+$ cells, and four had $3+$ cells. In two cases he described "mutton-fat" precipitates on the posterior surface of the detached vitreous humor".

\section{Retina}

Retinal signs observed on fundus examination include retinal vasculitis of large veins and papillitis and retinal disease is most clearly demonstrated by FA. Patients usually present with vitreous symptoms that include floaters and fuzzy vision, as well as 


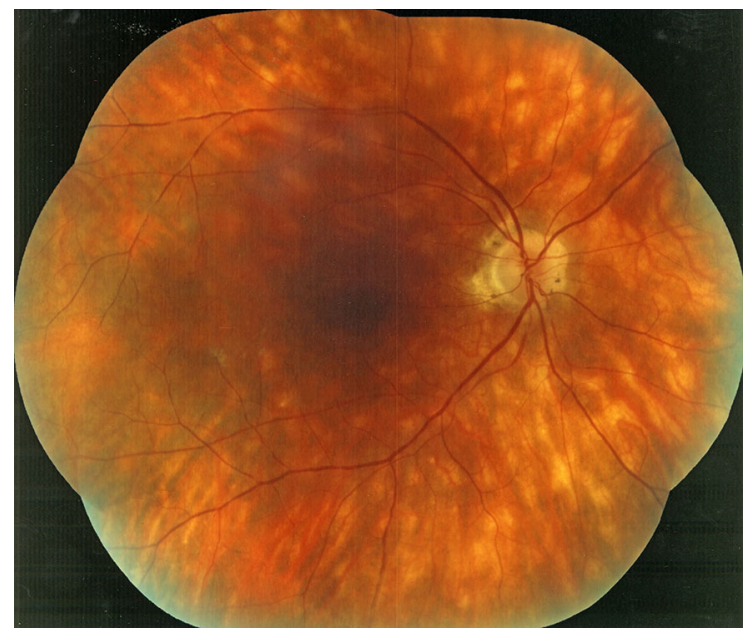

Fig. 2 Typical depigmented rice-shaped birdshot lesions (BRC fundus lesions) in a patient who declined treatment for 10 years

retinal symptoms such as dimness of vision, fluctuating vision, subjective scotomas, and peripheral vision difficulties. In the presence of vitritis, these symptoms lead the clinician to perform FA and OCT, which best illustrate retinal involvement (see below).

\section{Choroid}

Rice-shaped depigmented BRC fundus lesions were the hallmark sign that allowed the identification and discovery of this entity and turned out to be one of the main disease-defining criteria (Fig. 2). It was not possible to explore choroidal involvement in more detail at the time when the disease was initially described, as ICGA became available only in the early 1990s [12, 13]. Today, after the advent of ICGA and the histo-pathological findings reported by Gaudio et al. [7], it is clear that choroidal inflammation in BRC belongs to the category of primary stromal choroiditis, which also includes Vogt-Koyanagi-Harada (VKH) disease and sympathetic ophthalmia. Unlike these two latter conditions (which are purely primary stromal choroiditis), BRC is indeed a primary stromal choroiditis, but not only, as additional unrelated retinitis is also present. Choroidal ICGA lesions appear very early in the disease, but remain occult unless ICGA is performed or until BRC fundus lesions appear. Choroidal involvement is best demonstrated by ICGA (see below).
Disease process

In his report, Gass found many similarities between BRC and VKH disease and his comparisons were highly pertinent. The choroidal stroma is the site of inflammation in both diseases and stromal melanocytes are the target of the immune reaction. The immune reaction leads to depigmentation, which takes on the aspect of sunset glow fundus in VKH and the aspect of the rice-shaped depigmented BRC fundus lesions in BRC. Choroidal inflammation is characterized by primary stromal choroiditis in both diseases, meaning that the primary obligatory site of inflammation is located in the choroidal stroma [14]. The antigen that triggers the immune reaction to cause inflammation in the choroidal stroma has been determined in VKH, but is yet unknown for BRC [15].

On the other hand there are also significant differences between these two stromal choroiditis entities. The primary inflammatory process in $\mathrm{VKH}$ is limited to the choroidal stroma. It is a much more severe choroiditis that fills out the entire thickness of the choroid and in a second step causes significant secondary inflammation in neighboring structures such as the retina, vitreous compartment, and optic nerve head; this is the stage when the disease becomes clinically apparent [16]. BRC is a stromal choroiditis, but not only, as inflammatory events involve both the retina and the choroid in parallel and independently. Also, unlike in VKH, retinal inflammation in BRC is not the consequence of choroiditis. This is also the reason why aggressive treatment of $\mathrm{VKH}$ rapidly resolves inflammation in both compartments (which is not the case in BRC, in which retinal inflammation is more resistant because it is autonomous and not caused by spillover choroidal inflammation). Of this dual independent parallel inflammation, the choroidal response to treatment (as judged by the resolution of ICGA signs) is rapid and complete, while the response of retinal disease is much less satisfactory (Fig. 3) [17].

BRC should undoubtedly be classified as a granulomatous uveitis based clinically on the granulomatous KPs observed in 15-20\% of cases and the mutton-fat precipitates on the detached posterior vitreous described by Gass. Although the histopathology report by Gaudio et al. did not show clear granulomatous features it showed epithelioid cells in the infiltrates around the islets of stromal melanocytes. A subsequent histo-pathological report also failed to show a clear granulomatous histology. As indicated earlier the 
Fig. 3 Evolution of FA and ICGA scores over time. At presentation (stage 1), occult choroidal disease is maximal, but responds satisfactorily to treatment (measured by ICGA; green line). On the other hand, retinal disease is difficult to treat and is at best stabilized (yellow line)

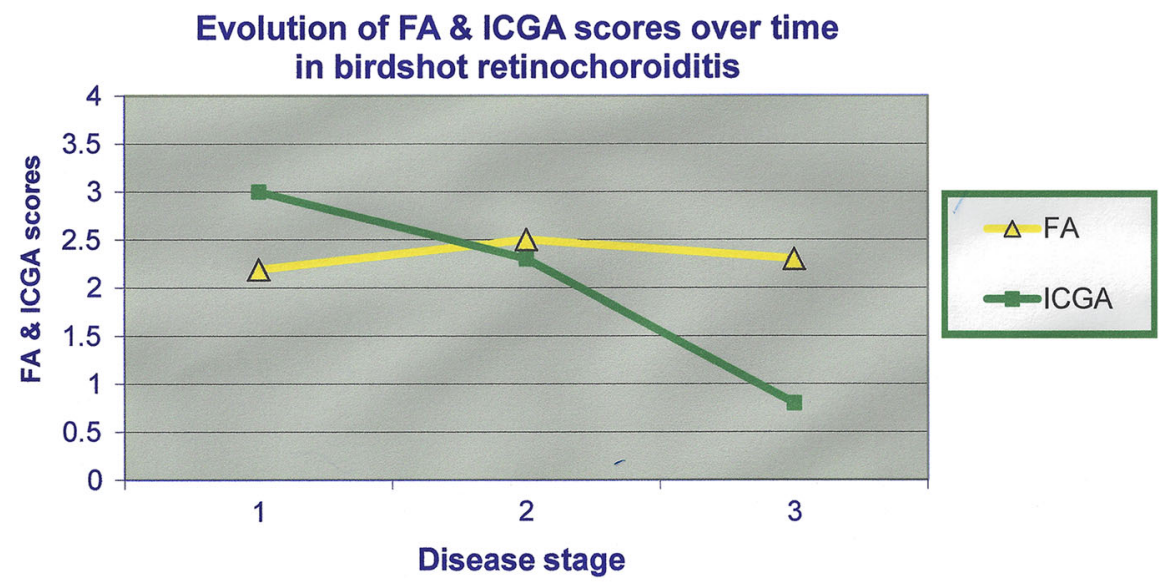

term of granulomatous uveitis is used here in its clinical sense.

The natural history of BRC is classically divided into an early stage and a late stage of disease. During the early stage, called the exudative stage, diffuse leakage from small and large retinal vessels causes diffuse retinal edema on FA and thickening on OCT. BRC fundus lesions may not yet be visible during this stage. During the later disease stages, choroidal BRC fundus lesions appear progressively and the retina evolves toward atrophy and thinning with thinned retinal vessels representing the atrophic stage of the disease. This evolution can be avoided to some extent if treatment is started early (see below).

\section{Diagnosis and functional assessment}

Fundus photography

Fundus photography is useful for following BRC fundus lesions and allows clinicians to document changes that occur between visits (Fig. 2).

\section{Fluorescein angiography}

BRC often presents with blurred vision and floaters due to vitreous infiltration associated with retinal vasculitis if FA is performed in these patients in the absence of any additional investigation (such as ICGA), the condition is often misdiagnosed as retinal vasculitis. The extent of retinal inflammatory involvement in BRC is best diagnosed using FA.
During the early exudative stage of BRC there are at least three characteristic signs that are observable using FA. In the early angiographic frames Gass (very accurately) observed "an increased retinal circulation delay". He also perspicaciously noted that in all patients "there was a varying degree of unexplained subnormal fluorescence of the retinal vessels during the entire course of the angiography" [3]. By performing dual FA/ICGA we showed that retinal circulation time is actually not increased; early ICGA frames show that large veins are marked by ICG within a normal time interval [5]. This FA pseudodelay is explained by the diffuse capillary exudation of fluorescein to the extent that there is not enough dye to normally mark the large veins. In contrast, the large ICG complex does not leak from retinal capillaries and normally marks large veins, which indicates that there is no real hemodynamic slowing (Fig. 4). Consequently, the first specific FA sign in BRC is massive and diffuse retinal capillary leakage with diffuse retinal edema, which was also described by Gass (Fig. 4).

This diffuse retinal edema also involves the macula, but strangely the foveal area appears to be spared often, which explains the good vision retained by a large proportion of patients for a relatively long period of time. In a recent study, we showed that true cystoid macular edema was not as frequent as indicated in the literature and that the central fovea might be devoid of leakage, even in cases with diffuse retinal and macular edema (Fig. 5) [18]. Although veins are often barely visible in early frames, in later frames there is leakage along large veins. This later leakage often leads to the 
Fig. 4 Pseudo-perfusion delay in retinal circulation. Early exudative stage of BRC showing absent fluorescein marking of large veins up to $39.94 \mathrm{~s}$. (bottom left and two top frames) after fluorescein injection. As shown on ICGA frame, this is not due to a perfusion delay as at $22 \mathrm{~s}$. ICG is already in the large veins. The cause of this pseudodelay is due to massive extrusion of the fluorescein molecule into the retina (top right picture)

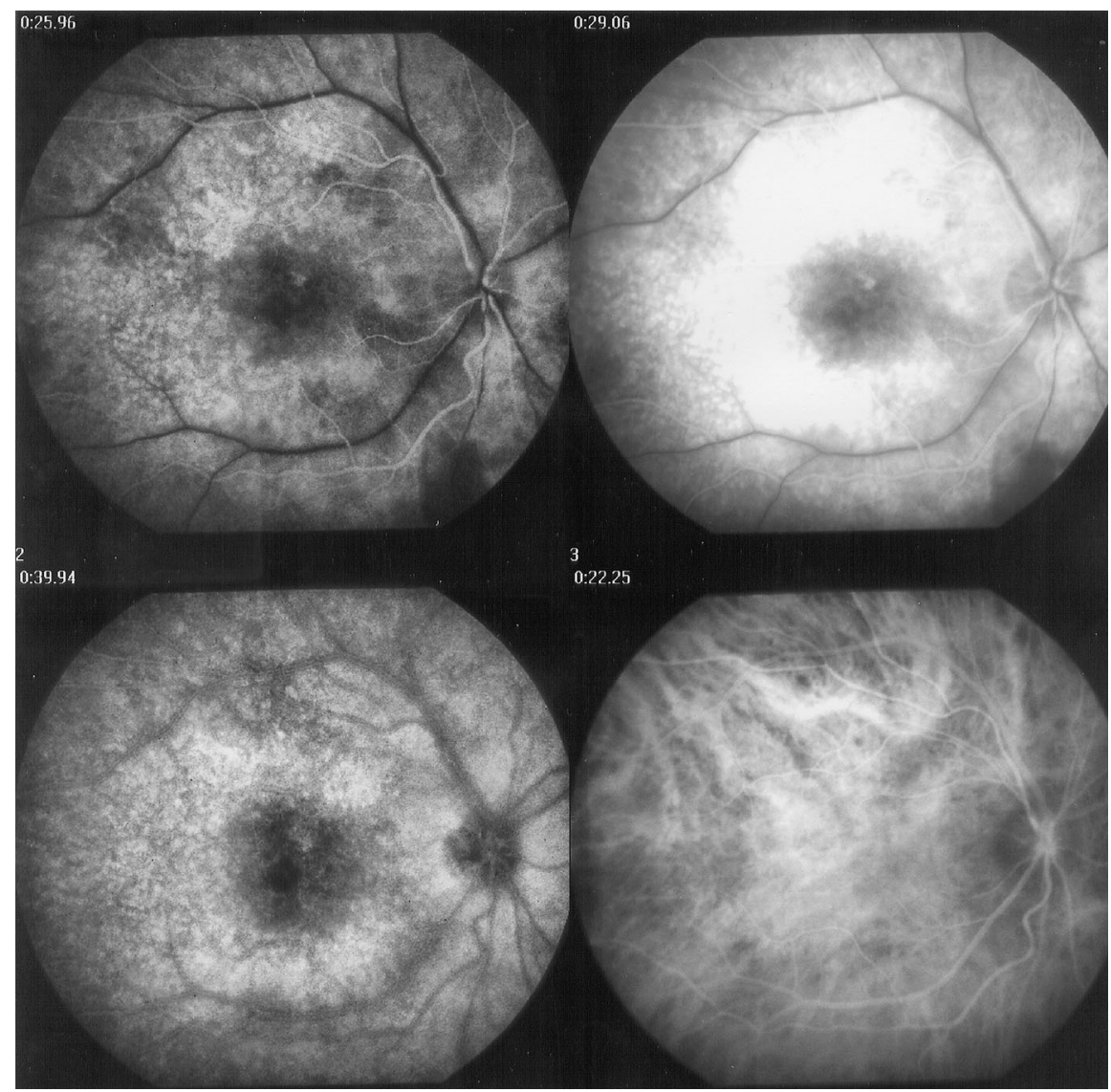

erroneous diagnosis of retinal vasculitis if ICGA is not performed, and this represents the second significant FA sign in BRC (Fig. 6). The third sign is disk hyperfluorescence, which is almost always present (Figs. 5, 6).

At a later stage of BRC, FA shows diffuse thinning of vessels including arteries and veins. Such thinning is a consequence of an extensive atrophic retinal process, which can be partially avoided by early and sustained inflammation suppressive treatment (IST) management.

\section{Optical coherence tomography (Fig. 7)}

Optical coherence tomography (OCT) allows another approach and provides a morphological explanation for the retinal signs observed using FA. We showed that in early disease a diffusely thickened retina was measured in the macula, but less so in the fovea, that showed less fluctuations during disease progression
(Fig. 8). This supports the FA finding showing that the fovea was relatively spared [19]. After a few years of disease progression, OCT revealed both areas of atrophy/thinning and areas of thickening, while in late disease the mean retinal thickness was reduced (mostly in patients for which treatment had been delayed) (Fig. 8). OCT also provided information about the vitreo-retinal interface; it revealed the presence of thin, pauci-contractile epiretinal membranes in up to $93 \%$ of cases [19].

The evolution of OCT from the exudative to the atrophic stage is shown on Fig. 8.

Indocyanine green angiography (Crucial for early diagnosis)

Indocyanine green angiography (ICGA) became available for clinical use in the early 1990s and enabled insight into choroidal inflammation [20]. One substantial advantage of this method is that it made 


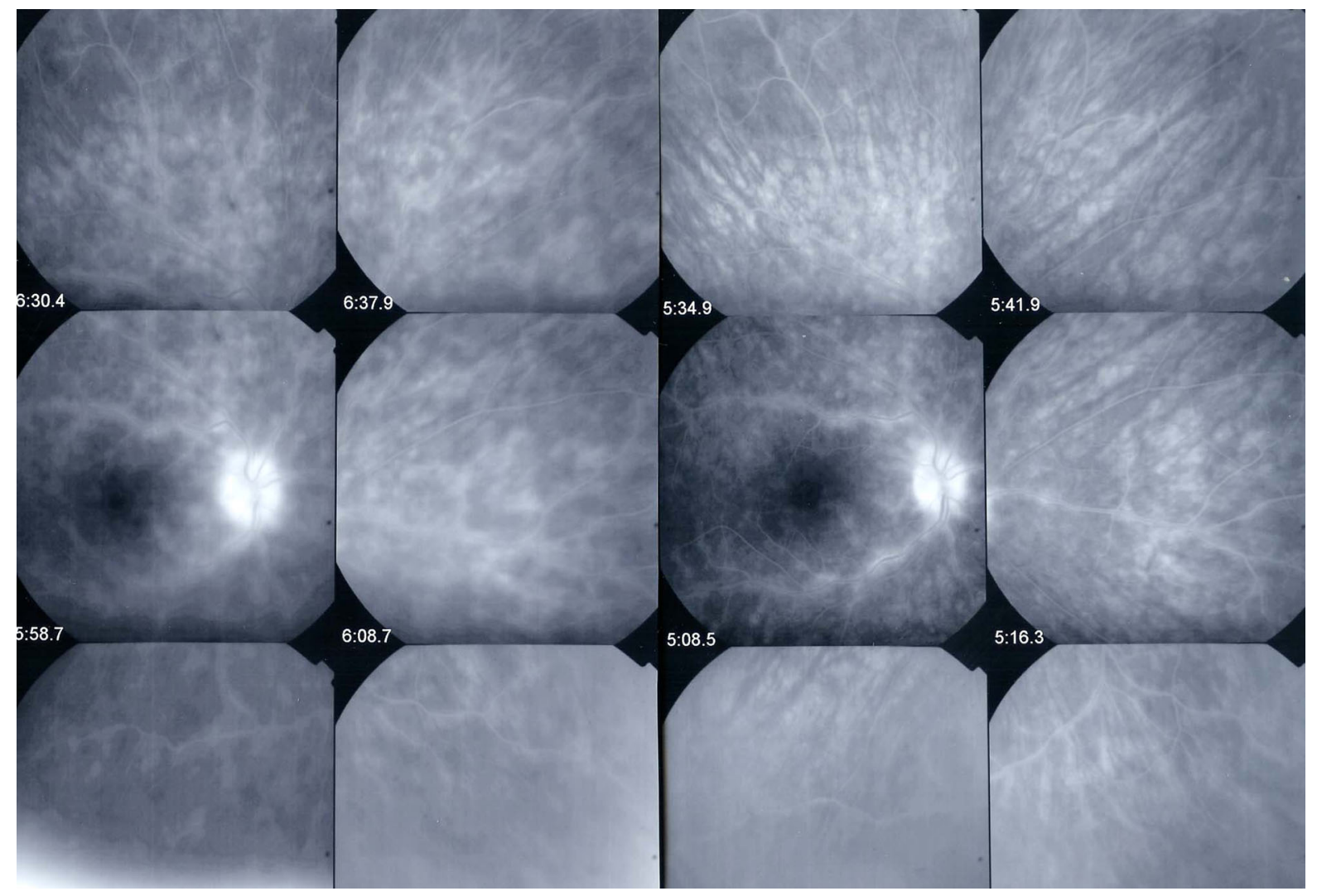

Fig. 5 FA signs, profuse retinal vasculitis and disk hyperfluorescence with relative sparing of fovea. Exudative stage FA panorama pictures of right eye exhibiting extensive vasculitis of small vessels and large veins as well as disk hyperfluorescence

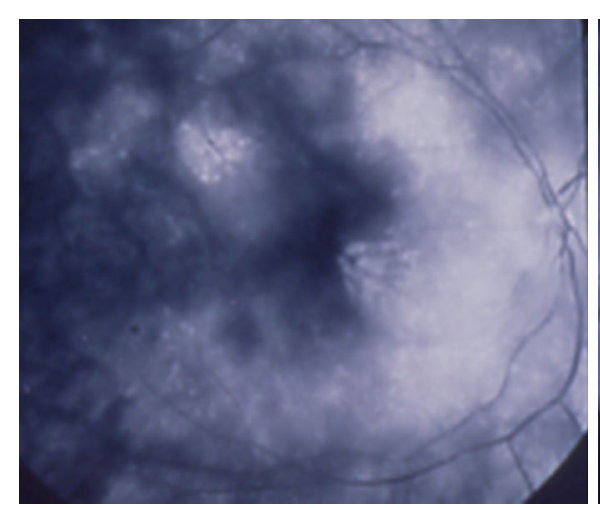

Fig. 6 Foveal sparing of diffuse retinal edema. This patient (same patient as in Fig. 6) was followed for more than 7 years for "neuroretinitis", without treatment. ICGA revealed numerous hypofluorescent dark dots bilaterally (not shown). There is (left sextet of pictures). Two months after introduction of IST treatment, retinal vasculitis regressed partially. (right sextet of pictures). Despite severe vasculitis the foveal area is relatively spared without obvious edema

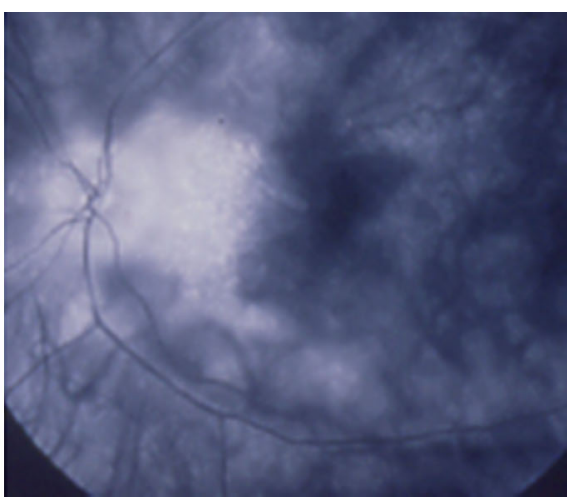

massive retinal edema that also involves the posterior pole, yet the fovea remains relatively spared, which explains the patient's preserved visual acuity 


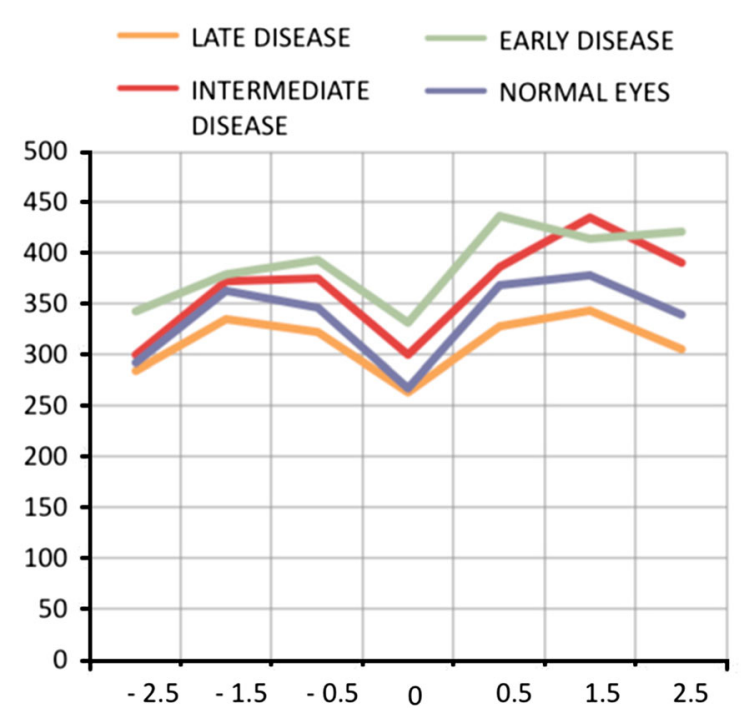

Fig. 7 Progression of retinal disease as followed by optical coherence tomography ( $x$ axis indicates distance from fovea $(=0) ; y$ axis indicates thickness of retina in microns). Early disease (green line) features thickening of the retina (and to a lesser degree the fovea). In late disease (yellow line) there is diffuse thinning except in the fovea; foveal thickness remains comparable to normals (purple line)
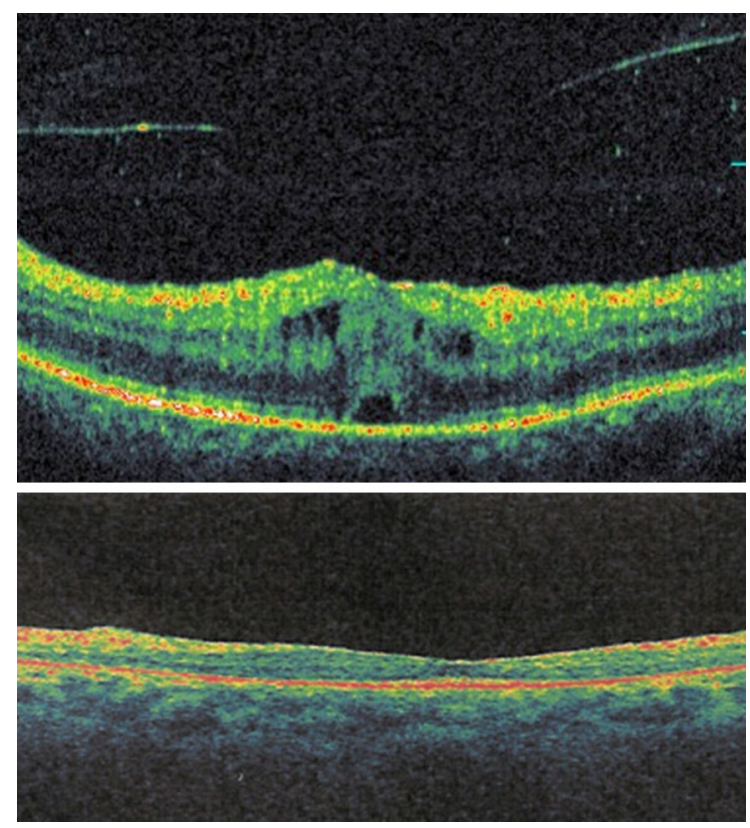

Fig. 8 OCT scans in early exudative stage and late atrophic stage of BRC. Top image shows a thickened retina in a BRC patient at presentation having presented vitreous symptoms for about 10 months. The bottom image shows a thinned retina in a patient who was treated after a few years of evolution possible the detection of occult choroidal lesions that were inaccessible when other investigational methods were used. Despite this undisputed advantage, many centers still do not use ICGA routinely [21]. ICGA allows clinicians to subdivide choroidal inflammation into choriocapillaritis entities (e.g., multiple evanescent white dot syndrome or acute posterior multifocal placoid pigment epitheliopathy) on one side and stromal choroiditis (e.g., VKH and BRC) on the other [22]. Despite its obvious advantage to the investigation of choroiditis, publications on ICGA in BRC are scarce. To date, of the 240 articles on BRC listed in PubMed, fewer than 10 publications have a strong focus on ICGA. We standardized ICGA findings in BRC in 1999 [23] and in 2011 we showed that ICGA is essential to establish an early diagnosis of BRC [24].

Relevant ICGA signs for diagnosis and disease monitoring are the presence of hypofluorescent dark dots (HDDs) and fuzziness of large choroidal vessels (Fig. 9). HDDs were suspected to be caused by stromal inflammatory foci; this hypothesis was confirmed histologically by Gaudio et al. [7]. Many HDDs become isofluorescent on late angiographic frames, which indicates that (unlike in VKH) lesions do not occupy the full thickness of the choroidal stroma; this possibility was also demonstrated by histopathology [23]. Fuzziness of choroidal vessels indicates vasculitis of large choroidal vessels [20]. ICGA signs are present before the appearance of characteristic BRC fundus lesions and ICGA is therefore essential for early diagnosis [24]. Most cases devoid of fundus lesions that were referred to us with vitritis and FA signs characteristic of BRC came with a diagnosis of retinal vasculitis. Presence of both ICGA angiographic signs including HDDs and fuzziness of vessels led to HLA testing, which confirmed the diagnosis when results were positive for the HLA-A29 antigen. Both signs respond promptly to the introduction of IST; therefore ICGA is an essential modality in monitoring choroidal disease.

In several reports HDDs are thought and reported to be the angiographic expression of BRC fundus lesions [25]. However, HDDs resolved in our treatment-naïve BRC patients having fundus lesions without affecting the size or number of these fundus lesions [18], likely because BRC fundus lesions correspond to areas of depigmentation that no longer have any active inflammation once the stromal pigment islets have been "digested". This explains why BRC fundus 


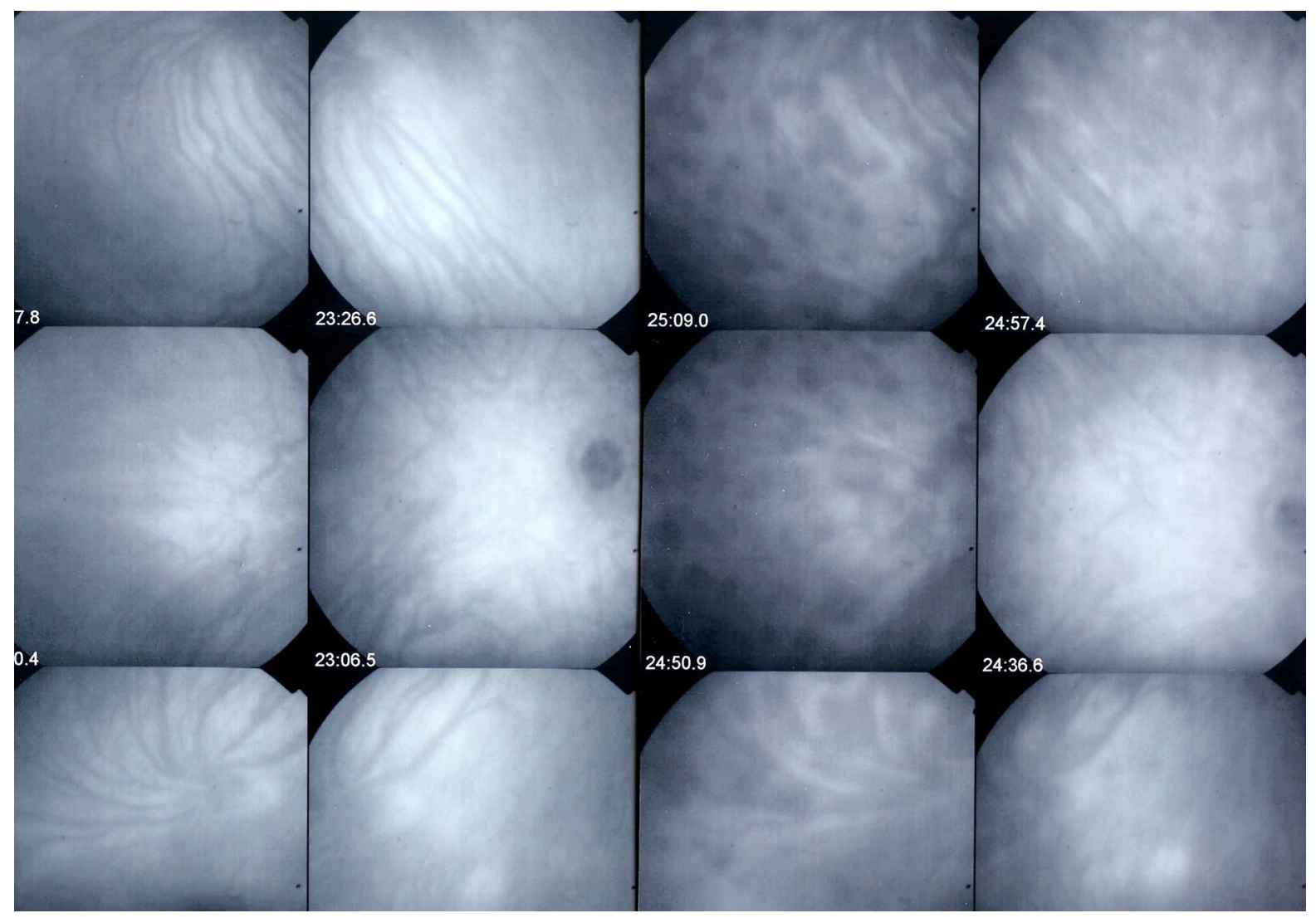

Fig. 9 ICGA signs in BRC (same patient as on Fig. 6). ICGA pictures in a patient complaining of vitreous symptoms since about 8-10 months (right sextet of frames). Numerous hypofluorescent dark dots (HDDs) can be seen and the choroidal vascular pattern is not recognized any more. After 2 months of

lesions are angiographically silent. Because it showed that patches of capillary leakage seen on FA, did not correspond to HDDs, ICGA also demonstrated that retinal inflammation was not related to choroidal inflammation, but occurred independently from choroidal inflammation (Fig. 10) [23].

Hypofluorescence is sometimes difficult to interpret in late disease because it can correspond to chorioretinal atrophy, in which case it will have an irregular form different from HDDs and will be hyperfluorescent on FA. Intrastromal fibrosis may be another origin of regular hypofluorescence resembling HDDs. Intrastromal fibrosis can impair ICG diffusion, and may thereby mimic disease activity. This is very rarely the case for BRC (in contrast to $\mathrm{VKH}$ ), because inflammation is milder and more limited for BRC than for VKH [26].
IST treatment, HDDs have disappeared and the choroidal vascular pattern is again visible (left sextet of frames). This case illustrates the relatively good response to therapy of the choroid, whereas the retinal involvement responds less readily as seen on Fig. 6

Visual field testing: a supporting diagnostic criterion

In most reports on BRC, visual acuity is the test used to monitor function. This does not seem to be appropriate because visual acuity can be preserved for a prolonged period, while dimness of vision and subjective visual field disturbance can be very pronounced. We showed in 1999 that this subjective visual disturbance was associated with visual field changes and that visual field monitoring allowed the assessment of treatment efficacy [27]. In a recent report, we showed that all patients diagnosed with BRC presented visual field disturbance, without exception [18]. One patient presented with bilateral tubular visual fields with a bilateral vision of 1.0 (20/ 20). This patient complained of severe peripheral field 
Fig. 10 Retinal

inflammation and choroidal inflammation are dual independent inflammatory events. The hypofluorescent dark dot encircled in the bottom left frame does not correspond to the fluorescein angiography (FA) hyperfluorescence that is visible in the same spot (top left frame.) Note, however, that the disk hyperfluorescence on FA (top right frame) also does not correspond to indocyanine green angiography (ICGA) hyperfluorescence (bottom right frame). This latter finding indicates that inflammation is not hyperacute in birdshot retinochoroiditis, in contrast to Vogt-Koyanagi-Harada disease, for which ICGA disk hyperfluorescence is often observed

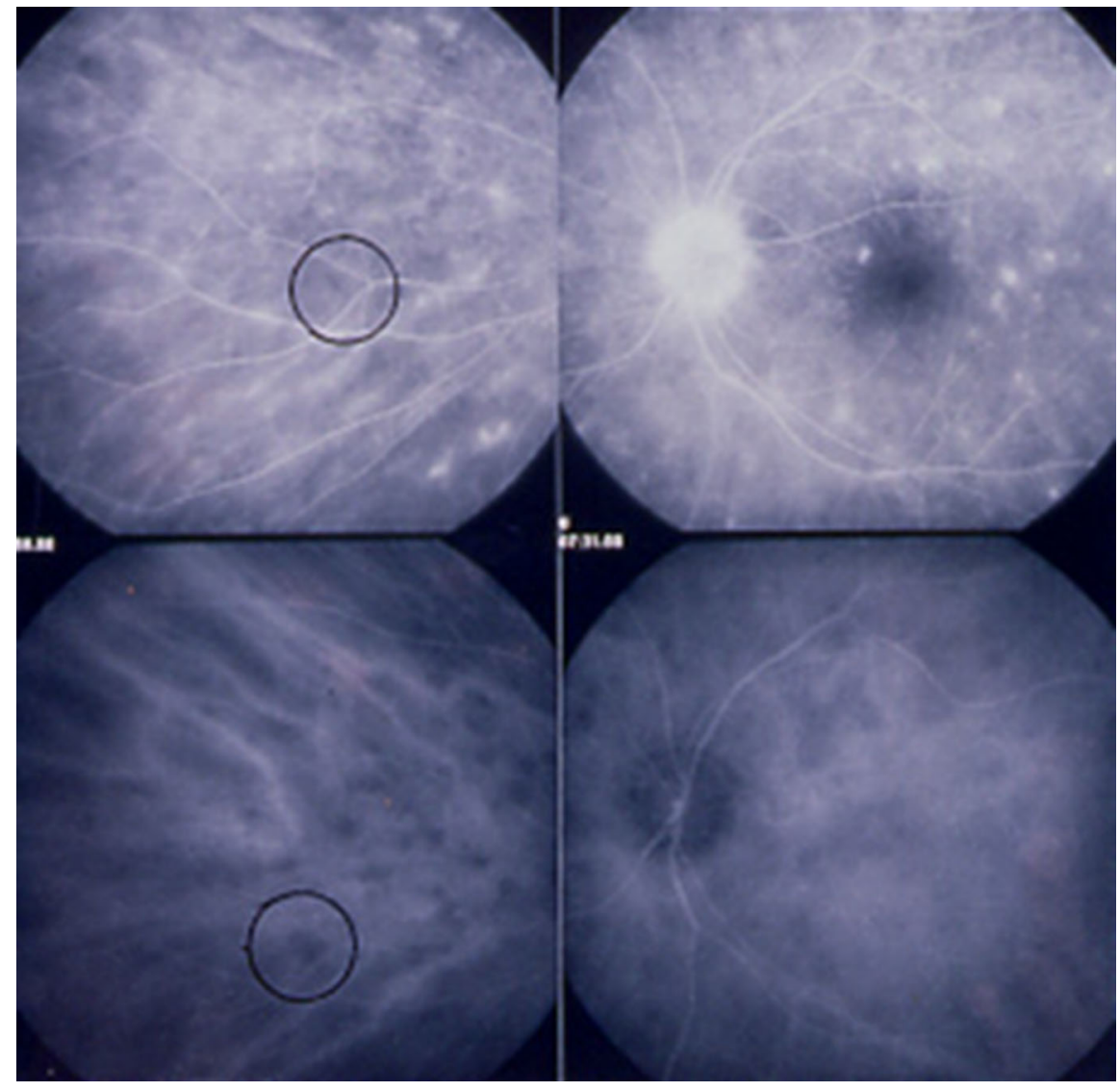

impairment that recovered substantially after introduction of IST (Fig. 11). The importance of visual field testing has been increasingly recognized in the last few years [28]. In one report, the rate of visual field disturbance amounted to $100 \%$, as in our series [29]. Non specific visual field alterations are common in many inflammatory conditions, especially in advanced stages. The presence of visual field changes in nearly $100 \%$ of patients might qualify this functional test not only for monitoring purposes but also as a supportive diagnostic criterion.

\section{Electrophysiology}

If available, electrophysiology is another investigational procedure that can be useful to localize disease impact and to monitor disease progression. In an early study on electroretinogram (ERG) in BRC, Priem et al. suggested that dysfunction resides in the inner retina, which corroborates FA and OCT findings that show diffuse retinal edema from diffusely leaking retinal capillaries (of which the functional counterpart is visual field changes) [30]. ERG data by Holder et al. also indicate that function of the inner retina is impaired [31]. These authors also showed that ERG improves after the initiation of treatment; it could therefore be considered a reliable method for monitoring treatment efficacy.

\section{HLA testing}

Association of BRC with the HLA-A29 tissue histocompatibility antigen was first reported in 1982 , 2 years after the entity was originally described [6]. The association rate was estimated to be approximately $95 \%$ in different subsequent reports [32, 33]. LeHoang et al. found that BRC was associated with the HLA-A29-2 subtype [34], which was later put in doubt [35]. It becomes increasingly likely that the association is closer to $100 \%$, as 


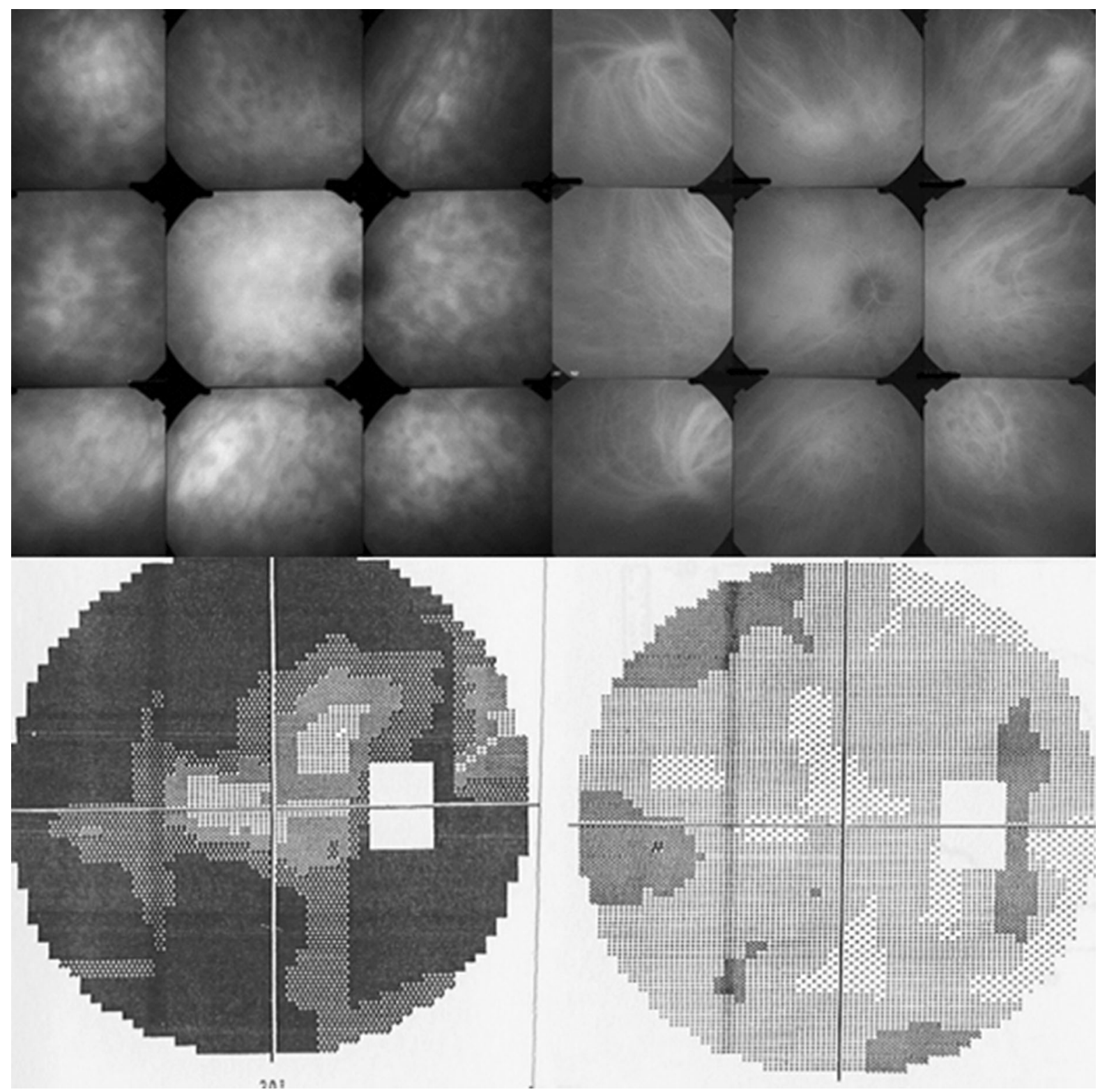

Fig. 11 Severe visual field changes without loss of visual acuity. This patient was seen 9 months after the onset of symptoms and complained of dimness of vision and subjective visual field impairment. Octopus ${ }^{\circledR}$ perimetry revealed tubular visual fields bilaterally (bottom left, right eye shown), severe retinitis on fluorescein angiography (not shown), as well as

false negatives are not so rare when the antibody testing method is used. The rate of false negative results can be reduced when PCR-based testing is used [36]. Truly HLA-A29-negative birdshot patients are a rarity if they exist at all and limiting the numerous hypofluorescent dark dots and fuzziness of choroidal vessels (top left set of 9 frames). After inflammation suppressive treatment (IST), the indocyanine green angiography choroidal aspect was restored to a quasi-normal condition (top right set of 9 frames) and the patient's visual field recovered substantially (bottom right, right eye shown)

diagnosis to PCR-positive HLA-A29 patients would exclude a tiny proportion of patients, if any patients at all. Therefore, presence of HLA-A29 antigen should be an essential criterion for the diagnosis of BRC allowing inclusion in clinical trials. 
Table 1 Global diagnostic criteria for birdshot retinochoroiditis (BRC)

1. Presence of vitritis in one or both eyes (required)

2. Presence of retinal vasculitis in one or both eyes (required)

3. Stromal choroiditis, as evidenced by ICGA, in both eyes (required)

4. HLA-A29 antigen positivity (required)

5. Visual field anomalies in one or both eyes (supportive)

6. Absence of extra-ocular inflammatory site (supportive)

7. Presence of rice-shaped depigmented "birdshot lesions" (BRC fundus lesions) (strongly supportive but not required)

\section{Diagnostic criteria (the need for appropriate criteria)}

In 2006 a group of experts published research criteria for the diagnosis of BRC [4]. However, these criteria fail to truly characterize BRC, especially early stage disease. The major shortcomings are the omission of ICGA signs, which are present in nearly $100 \%$ of cases; lack of any reference to the visual field changes that are present in almost $100 \%$ of cases; the characterization of HLA-A29 antigen as merely a supporting factor even though it is present in almost $100 \%$ of cases; and finally the lack of acknowledgment that KPs cannot be an exclusion criterion as they are present in close to $20 \%$ of treatment-naïve patients. There is an urgent need for new and proper criteria for the correct appraisal and management of BRC. In our studies, we used following criteria: presence of vitritis and retinal vasculitis in one or both eyes, visual field anomalies in one or both eyes, stromal choroiditis as evidenced by ICGA in both eyes, HLA-A29 antigen positivity and absence of extra-ocular inflammation sites. An additional (but not obligatory) criterion was the presence of rice-shaped, depigmented "birdshot lesions" (BRC fundus lesions) (Table 1).

\section{Therapy \& evolution}

\section{Treatment rationale}

For many years after BRC was described, the opinion prevailed that BRC did not necessarily require aggressive IST [37-39]. The reason for such a position was twofold: (1) early reports had placed the efficacy of IST in doubt and (2) the functional criterion used to follow disease evolution was visual acuity, which we now know to be inappropriate because central vision remains excellent in a substantial proportion of patients, even after years of disease progression [40].

There is increasing evidence that aggressive and sustained treatment is probably needed in the majority of patients; [41, 42] not more than 10-15\% of patients present a relatively mild course [8]. Once the diagnosis is established, our criterion to treat is the occurrence of visual field defects. If involvement is unilateral, then the introduction of systemic immunosuppressive treatment can sometimes be deferred for a period of time using sub-Tenon's injections of triamcinolone acetonide (40 mg per injection) every 4-6 months if the other eye is not functionally affected. Once systemic treatment is decided, our regimen combines systemic or sub-Tenon's steroids with immunosuppressants, mostly mycophenolic acid $\left(\right.$ Myfortic $^{\circledR}$ ). Steroids are subsequently tapered to 0 or to $<7.5 \mathrm{mg}$ per day. In case of insufficient recovery of visual fields and/or persistence of retinal inflammatory signs (mainly monitored by FA), a second immunosuppressant or biologic agent is added (e.g., anti-TNF$\alpha$ antibodies). We demonstrated that substantial improvement of visual field parameters and maintenance of central vision can be obtained, as well as resolution of ICGA signs and improvement of FA signs [18]. Early treatment is usually the rule and has been deemed necessary in up to $90 \%$ of cases. However, at present, treatment is too often delayed because of a delayed diagnosis or because patients decline treatment. We showed that insufficient or delayed treatment results in retinal atrophy, as evidenced by OCT [19].

Phenotype of BRC is modified by early and sustained therapy

We recently showed that, among patients that were treated before the development of BRC fundus lesions, the appearance of such lesions could be prevented in 5 of 6 patients treated within a mean of 6.5 months of symptoms and observed during a mean period of 10 years of treatment [8]. In this regard BRC behaved like VKH, another stromal choroiditis in which it has also been shown that early, vigorous, and prolonged treatment can prevent the development of sunset glow 
a

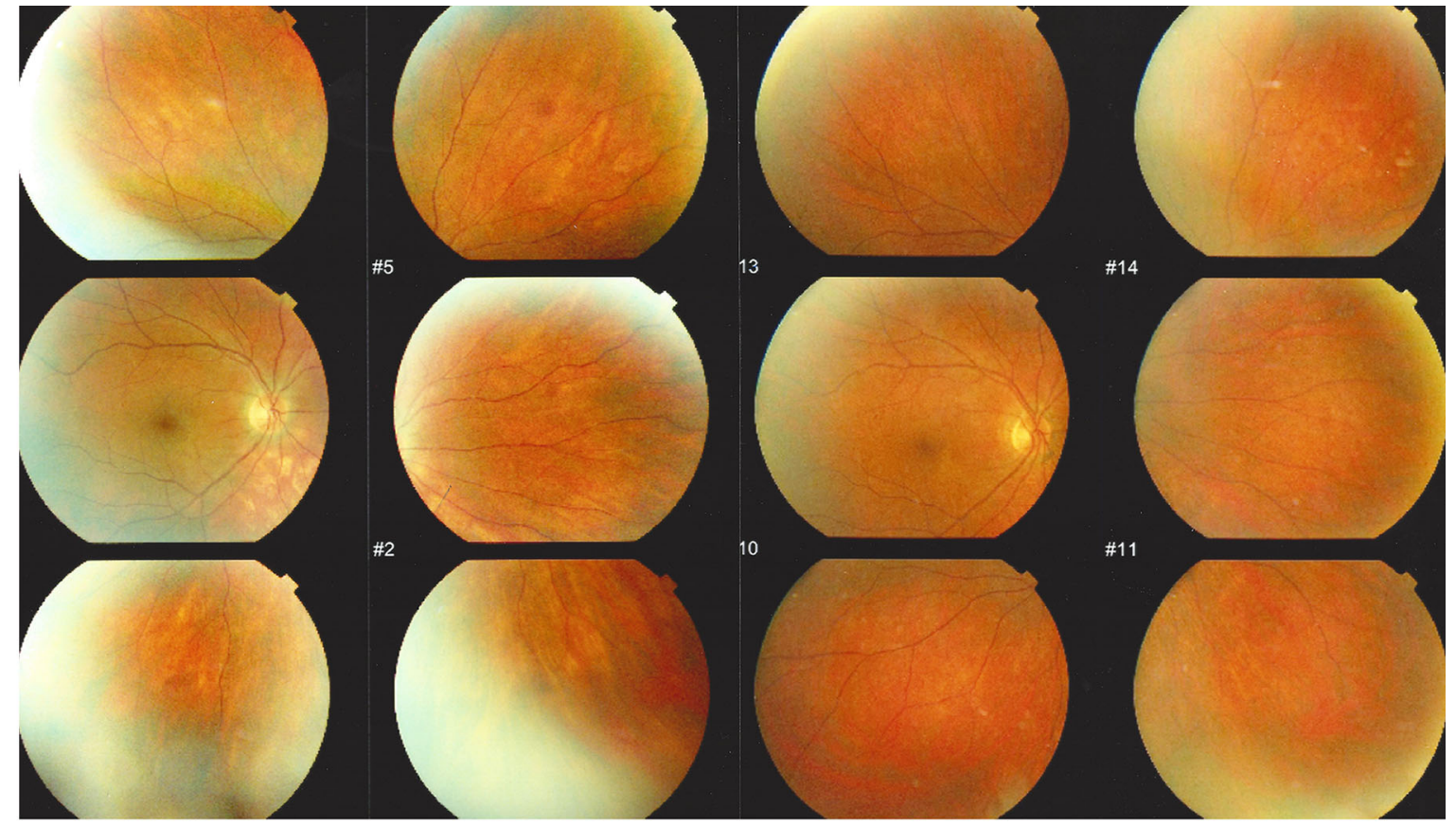

b

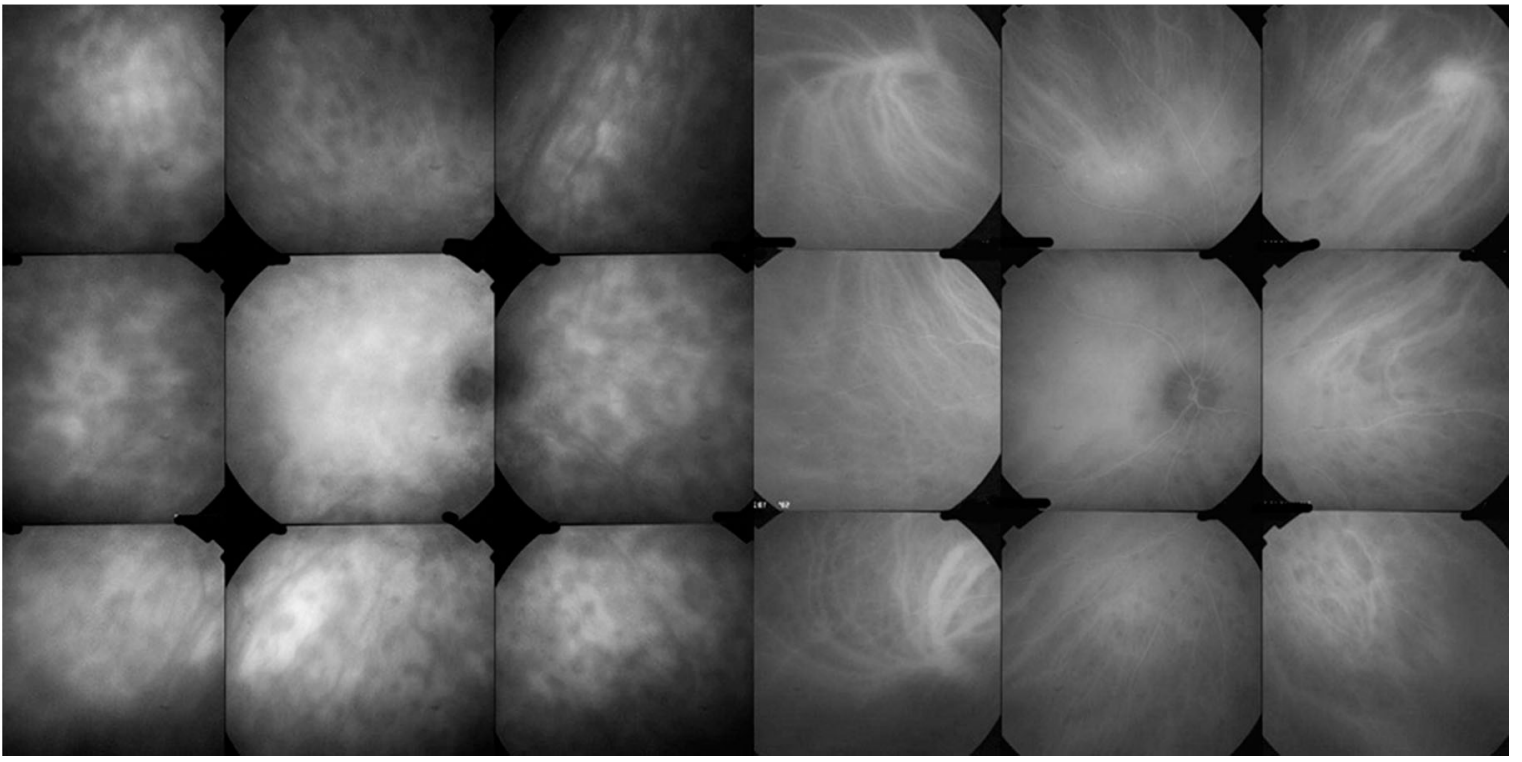

Fig. 12 This female patient, aged 54 years, was referred 9 months after the onset of ocular symptomatology consisting of dimness of vision and myodesopsias. She was diagnosed as BRC based on her FA and ICGA findings, her tubular visual fields, and positive HLA-A29 antigen. Despite relatively early diagnosis, fundus examination already revealed typical BRC fundus lesions (Fig. 12a, left sextet of images) associated with the usual FA signs of vasculitis of the large retinal veins, diffuse retinal exudation/leakage from the small capillaries, and disk hyperfluorescence. ICGA showed extensive choroidal involvement, with many HDDs and fuzziness of the choroidal vessels (Fig. 12b, left sextet of images). IST maintenance therapy consisted of azathioprine $(2.0 \mathrm{mg} / \mathrm{kg})$ and low-dose prednisone after tapering from an initial dose of $1 \mathrm{mg} / \mathrm{kg}$. After 13 years of treatment, not only did the ICGA signs resolve completely (Fig. 12b, right sextet of images), but the BRC fundus lesions resolved as well (Fig. 12a, right sextet of images) 
fundus (Fig. 12) [43]. Indeed, such prevention of BRC fundus lesions requires early diagnosis, which is not possible with the present diagnostic criteria.

\section{Conclusions}

BRC is a uveitis of the granulomatous type (used in its clinical sense). It appears to lack any known extra-ocular site of inflammation and demonstrates nearly $100 \%$ association with the leukocyte histocompatibility antigen HLA-A29. Dual parallel non-related inflammation of the retina and the choroid is particular to BRC. Retinal involvement is responsible for substantial clinical morbidity if the disease is left untreated. Treatment (IST) must be started early and should be vigorous and prolonged. Choroidal involvement responds rather well and rapidly to IST; the development of depigmented BRC fundus lesions can even be prevented if treatment is started early. The impact of therapy on retinal disease is more limited but can likely avoid retinal atrophy if treatment is applied early. However, ICGA is crucial to achieve early diagnosis before the appearance of what has thus far been considered the hallmark of BRC: riceshaped choroidal depigmented fundus lesions.

\section{References}

1. Gasch AT, Smith JA, Whitcup SM (1999) Birdshot retinochoroidopathy. Br J Ophthalmol 83:241-249

2. Ryan SJ, Maumenee AE (1980) Birdshot retinochoroidopathy. Am J Ophthalmol 89:31-45

3. Gass JDM (1981) Vitiliginous chorioretinitis. Arch Ophthalmol 99:1778-1787

4. Levinson RD, Brezin A, Rothova A et al (2006) Research criteria for the diagnosis of birdshot chorioretinopathy: results of an international consensus conference. Am J Ophthalmol 141:185-187

5. Guex-Crosier Y, Herbort CP (1997) Prolonged retinal arterio-venous circulation time by fluorescein but not by indocyanine green angiography in birdshot chorioretinopathy. Ocul Immunol Inflamm 5:203-206

6. Nussenblatt RB, Mittal KK, Ryan S et al (1982) Birdshot retinochoroidopathy associated with HLA-A29 antigen and immune responsiveness to retinal S-antigen. Am J Ophthalmol 94:147-158

7. Gaudio PA, Kaye DB, Crawford JB (2002) Histopathology of birdshot retinochoroidopathy. $\mathrm{Br} \mathrm{J}$ Ophthalmol 86:1439-1441

8. Papadia M, Knecht PB, Herbort CP. (2013) Early and sustained treatment modifies the phenotype of birdshot retinochoroiditis. Acta Ophthalmol 91( s252). doi: 10.1111/ j.1755-3768.2013.x

9. Knecht PB, Papadia M, Herbort CP (2013) Granulomatous keratic precipitates in birdshot retinochoroiditis. Int Ophthalmol 33:133-137

10. Guex-Crosier Y, Pittet N, Herbort CP (1995) Sensitivity of laser flare photometryto monitor inflammation in uveitis of the posterior segment. Ophthalmology 102:613-621

11. Monnet D, Brézin A, Holland GN et al (2006) Longitudinal cohort study of patients with birdshot chorioretinopathy. I. Baseline clinical charcteristics. Am J Ophthalmol 141:135-142

12. Herbort CP, LeHoang P, Guex-Crosier Y (1998) Schematic interpretation of indocyanine green angiography in posterior uveitis using a standard angiographic protocol. Ophthalmology 105:432-440

13. Herbort CP (1998) Posterior uveitis: new insights provided by indocanine green angiography. Eye 12:757-759

14. Bouchenaki N, Herbort CP (2004) Stromal choroiditis. In: Pleyer U, Mondino B (eds) Essentials in ophthalmology: uveitis and immunological disorders. Springer, Berlin, pp 234-253

15. Sugita S, Takase H, Taguchi C et al (2006) Ocular infiltrating CD4+ T cells from patients with Vogt-KoyanagiHarada disease recognize human melanocyte antigens. Invest Ophthalmol Vis Sci 47:2547-2554

16. Herbort CP, Mantovani A, Bouchenaki N (2007) Indocyanine green angiography in Vogt-Koyanagi-Harada disease: angiographic signs and utility in patient follow-up. Int Ophthalmol 27:173-182

17. Herbort CP, Probst K, Cimino L, Tran VT (2004) Differential inflammatory involvement in retina and choroid in birdshot chorioretinopathy. Klin Monbl Augenheilkd 221:351-356

18. Papadia M, Herbort CP (2013) Reappraisal of birdshot retinochoroiditis (BRC): a global approach. Graefe's Arch Clin Exp Ophthalmol 251:861-869

19. Papadia M, Jeannin B, Herbort CP (2012) OCT findings in birdshot chorioretinitis: a glimpse into retinal disease evolution. Ophthalmic Surg Lasers Imaging 43(6 suppl):S25S31

20. Herbort CP, Mantovani A, Papadia M (2012) Use of indocyanine green angiography in uveitis. Int Ophthalmol Clin 52:13-31

21. Herbort CP, Neri P, Abu El Asrar AA et al (2012) Is ICGA still relevant in inflammatory eye disorders? Why this question has to be dealt with separately from other eye conditions. Retina 32:1701-1703

22. Herbort CP, Papadia M, Mantovani A (2012) Classification of choroiditis based on inflammatory lesion process rather than fundus appearance: enhanced comprehensionthrough the ICGA concepts of the iceberg and jellyfish effects. Klin Monbl Augenheilk 229:306-313

23. Fardeau C, Herbort CP, Kullmann N et al (1999) Indocyanine green angiography in birdshot chorioretinopathy. Ophthalmology 106:1928-1934

24. Papadia M, Herbort CP (2012) Indocyanine green angiography (ICGA) is essential for the early diagnosis of birdshot chorioretinopathy. Klin Monbl Augenheilkd 229:348-352

25. Leder HA, Galor A, Thorne JE, Jabs D (2008) Disappearance of classic birdshot spots after immunosuppression with 
tacrolimus and mycophenolate mofetil. Br J Ophthalmol 92:291

26. Knecht PB, Mantovani A, Herbort CP (2013) Indocyanine green angiography-guided management of Vogt-Koyanagi-Harada disease. Int Ophthalmol 33:571-577 Jan 1 (Epub ahead of print)

27. de Courten C, Herbort CP (1998) Potential role of computerized visual field testing for the appraisal and follow-up of birdshot chorioretinopathy. Arch Ophthalmol 116:1389-1391

28. Thorne JE, Jabs DA, Kedhar SR et al (2008) Loss of visual field among patients with birdshot chorioretinopathy. Am J Ophthalmol 145:23-28

29. Gordon LK, Monnet D, Holland GN et al (2007) Longitudinal cohort study of patients with birdshot chorioretinopathy IV. Visual field. Am J Ophthalmol 144:829837

30. Priem HA, De Rouck A, De Laey JJ, Bird AC (1988) Electrophysiologic studies in birdshot chorioretinopathy. Am J Ophthalmol 106:430-436

31. Holder GE, Robson AG, Pavésio C, Graham EM (2005) Electrophysiological characterization and monitoring in the management of birdshot chorioretinopathy. Br J Ophthalmol 88:709-718

32. Baarsma GS, Priem HA, Kijlstra A (1990) Association of birdshot retinochoroidopathy and HLA-A29 antigen. Curr Eye Res 9(Suppl 1):63-68

33. Brezin AP, Monnet D, Cohen JH, Levinson RD (2011) HLA-A29 and birdshot chorioretinopathy. Ocul Immunol Inflamm 19:397-400

34. LeHoang P, Osdemir N, Benhamou A et al (1992) HLAA29.2 subtype associated with birdshot retinochoroidopathy. Am J Ophthalmol 113:33-35
35. Levinson RD, Rajalingam R, Park MS et al (2004) Human leukocyte antigen A29 subtypes associated with birdshot retinochoroidopathy. Am J Ophthalmol 138:631-634

36. Wender JD, Fu AD, Jumper JM et al (2008) False-negative antibody-based HLA-A29 typing in two patients with birdshot chorioretinopathy. Br J Ophthalmol 92:1153-1154

37. Priem HA, Oosterhuis JA (1988) Birdshot chorioretinopathy: clinical characteristics and evolution. Br J Ophthalmol 72:646-659

38. The American academy of ophthalmology basic and clinical science course, The Foundation of the American Academy of Ophthalmology, San Francisco (Pub.), Volume 9, Intraocular Inflammation and uveitis, 2000-2001 Edition, pp 170-1

39. Nussenblatt RB, Wuitcup SM, Palestine AG. Birdshot retinochoroidopathy. In Uveitis, fundamentals and clinical practice, 2nd edition. Mosby, St.-Louis 1996; pp 325-333

40. Rothova A, Berendschot TTJM, Probst K et al (2004) Birdshot chorioretinopathy, long-term manifestations and visual prognosis. Ophthalmology 111:954-959

41. Kiss S, Ahmed M, Leiko E, Foster CS (2005) Long-term follow-up of patients with birdshot retinochoroidopathy treated with corticosteroid-sparing systemic immunomodulatory therapy. Ophthalmology 112:1066-1071

42. Cervantes-Castaneda RA, Gonzalez-Gonzalez LA, Cordero-Coma M et al (2013) Combined therapy of cyclosporine A and mycophenolate mofetil for the treatment of birdshot retinochoroidopathy: a 12-month follow-up. Br J Ophthalmol 97:637-643

43. Bouchenaki N, Herbort CP (2011) Indocyanine green angiography guided management of Vogt-Koyanagi-Harada disease. J Ophthalmic Vis Res 6:241-248 